\title{
Radical Scavenging Activities of Plant Food of Alkyl-oxy and Superoxide Radicals
}

\author{
Mitsuko Ukai $^{1 *}$, Hiromi Kameya ${ }^{1}$, Hideo NAKAmura ${ }^{1}$ and Yuhei Shimoyama ${ }^{2}$ \\ ${ }^{1}$ Department of Environmental Science, Hokkaido University of Education, 1-2 Hachiman-cho, Hakodate 040-8567, Japan \\ ${ }^{2}$ Soft-Matter Physics Laboratory, Graduate School of Emergent Functional Sciences, Muroran Institute of Technology, 27-1 Mizumoto- \\ cho, Muroran 050-8585, Japan
}

Received June 26, 2009; Accepted July 30, 2009

The spin trapping ability against alkyl-oxy and superoxide radicals was examined using a new reagent 5-(2,2-dimethy-1,3-propoxycyclophosphoryl)-5-methyl-1-pyrroline N-oxide (CYPMPO), a colorless crystalline that is very soluble in aqueous solution. For careful detection of spin adducts, we used borosilicate ESR flat cells. We examined plant specimens from teas, vegetables and fruits. CYPMPO successfully trapped alkyl-oxy and superoxide radicals in the plant extracts. We generated very pure alkyl-oxy radicals by illuminating phosphate buffer solution containing 2,2'-azobis(2-amidinopropane) dihydrochloride (AAPH) and CYPMPO with Hg-Xe arc lamp. Superoxide radicals were similarly generated using hypoxanthine (HPX), diethylene-triamine-pentaacetic acid (DTPA), CYPMPO and xanthenes oxidase (XOD). The spin adducts by CYPMPO were sensitive and very stable. The trapping activity for the alkyl-oxy and superoxide radicals was measured by the 2-amino-5-\{[2-[(carboxymethyl)amino]-1-(mercaptomethyl)-2oxoethyl]amino\} 5-oxopentanoic acid (GSH) and superoxide dismutase (SOD) equivalents, respectively. Thus, CYPMPO is useful for its high capability of radical trapping.

Keywords: electron spin resonance, radical trapping, alkyl-oxy radical, superoxide radical, plant extracts

\section{Introduction}

Antioxidants are ubiquitous in almost all plants. They play roles as efficient scavengers for radicals (MorenoSánches, 2002). Oxidation processes are crucial for food storage. Oxidation is the major cause of chemical spoilage of food, leading to rancidity and/or deterioration of the nutritional quality, color, flavor and texture (Percival, 1996). Thus, total antioxidant capacity is an important factor for evaluating food quality.

A highly complex antioxidant protection mechanism has evolved to protect body organ systems against reactive oxygen species. This mechanism involves a variety of endogenous and exogenous components to eliminate free radicals. These components include nutrient-derived antioxidants, antioxidant enzymes, metal-binding proteins, and numerous other antioxidant phytonutrients present in a wide variety of plant foods (Percival, 1996).

Basic methods to measure antioxidant capacity in foods have been reviewed by Prior et al. (2005). Three assays are

*To whom correspondence should be addressed.

E-mail: mitsuko@hak.hokkyodai.ac.jp recommended for standardization, oxygen radical absorbance capacity (ORAC), the Folin-Ciocalteu method and the 6-hydroxy-2,5,7,8-tetramethylchroman-2-carboxylic acid (Trolox) equivalent antioxidant capacity method. Methods of analysis of water soluble and lipid soluble antioxidant capacity in foods were extended by the modifications of the ORAC procedure (Prior et al., 2003). These methods have been compiled into the USDA food database of antioxidant capacity of foods.

The antioxidant capacity of extracts from some herbs has been reported by means of the ORAC assay (Zheng et al., 2001). Hydrogen peroxide- and hypochlorous acid-scavenging activities have been reported on Mediterranean spices (Martinéz et al., 2001). The hydroxyl radical scavenging ability using the deoxyribose assay has also been reported on the antioxidant activity of certain Mediterranean spices (Martinéz et al., 2001), as well as that of extracts from onion skin (Suh et al., 1996) and garlic (Yang et al., 1994). The antioxidant activity of phenolic compounds from sage and basil were determined using the cation radical 2,2'-azinobis(3ethylbenzthiazoline-6-sulphonate) (ABTS) (Jun et al., 2001).

The ability to scavenge the free radical 2,2-diphenyl- 
1-picrylhydrazyl (DPPH) is considered to be an easy method for evaluating scavenging activity of antioxidants. DPPH is relatively stable, and commercially available. The DPPH assay has been reported to characterize antioxidant activity of quercetin from onion skin (Suh et al., 1996), polyphenol from sage (Jun et al., 2001), and extracts from many kinds of spices (Wang et al., 1998; Kikuzaki et al., 1994; Exarchou et al., 2002). In contrast, superoxide anion radical scavenging activity of crude extracts from oregano has been determined by a lactate dehydrogenase oxidation system (Horváthová et al., 2007).

Electron spin resonance (ESR) spectroscopy is a powerful technique for free radical evaluation (Polovka et al., 2007). As it allows radicals to be detected in dry samples, ESR spectroscopy is very useful for identifying radicals in irradiated food (Delincee, 2002), a new approach for detecting the irradiation history (Yordanov and Aleksieva, 2004). This technique has been applied to radiation-induced radicals in black pepper (Ukai and Shimoyama, 2003), oregano (Exarchou et al., 2002), parsley (Ukai et al., 2008) and crude Chinese medicines (Nakamura et al., 2006). ESR spectroscopy can also detect radicals in liquid media, including the hydroxyl radical scavenging ability of spices (Suhaj et al., 2006). Radical scavenging activities of spices are reviewed by Suhaj (2006).

In the present study, we employed a new and stable spin trap reagent CYPMPO (Kamibayashi et al., 2006) to determine the radical scavenging activity in plant extracts. Using CYPMPO, we developed a new trapping protocol for alkyl-oxy radicals in several plant extracts that are known to possess high antioxidant activities. The trapping activity of superoxide radicals by CYPMPO was also described in plant extracts.

\section{Materials and Methods}

Reagents The spin trap CYPMPO (Fig. 1) was from Radical Research (Hino, Japan). All other chemicals were from Wako Pure Chemical Co. (Tokyo, Japan) and were of the highest grade.

Samples The plant foods in this study include teas (agaricus tea, refined green tea, and roasted green tea), vegetables (ginseng, Japanese basil, burdock, green pepper, parsley, green asparagus, spinach, tomato, shiitake mushroom, and sweet potato) and fruits (blue berry, huscup, and aronia).

Samples $(1 \mathrm{~g})$ were prepared by dissolving in pure water or $80 \%$ ethanol for determining their trapping activities. All samples were freshly prepared. The samples were soaked in $30 \mathrm{~mL}$ extraction solution (pure water or $80 \%$ ethanol) and incubated at $4{ }^{\circ} \mathrm{C}$ for $24 \mathrm{~h}$. After filtration with filter paper, extracts were adjusted to $50 \mathrm{~mL}$ with the addition of the<smiles>N[C@@H](CCC(=O)N[C@@H](CS)C(=O)NCC(=O)O)C(=O)O</smiles>

$\mathrm{GSH}$

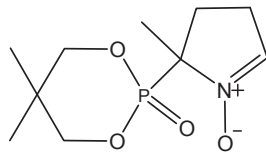

CYPMPO

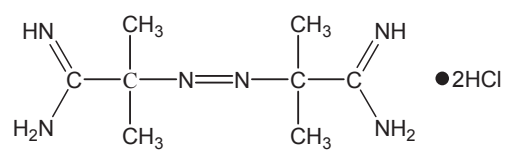

$\mathrm{AAPH}$
Fig. 1. The structure of CYPMPO, AAPH and GSH.

same solution. These extracts were frozen.

Alkyl-oxy radical trapping activity A light illuminator (cat. No. RUVF-203SR, Radical Research) was employed to produce free radicals from AAPH (Fig. 1). The light source was a $200 \mathrm{~W}$ Hg-Xe arc lamp (Sanei Electronics, Tokyo, Japan), which delivers mainly UV light with a wavelength of 200 to $400 \mathrm{~nm}$. The illuminator was equipped with a quartz fiber optical guide, and its far end was mechanically fitted to the hole in front of the ESR cavity. The illuminator is equipped with a computer controllable mechanical shutter, and the illumination period can be set in the range of 0.1 to $100 \mathrm{~s}$ with a precision of $0.01 \mathrm{~s}$. The illumination condition was optimized so that AAPH was efficiently photodecomposed to generate free radicals (Krainev et al., 1996). Using the attenuated $\mathrm{Hg}$-Xe arc lamp light, $80 \mu \mathrm{L}$ of phosphate buffer solution (100 mM, pH 7.4), containing $50 \mu \mathrm{L}$ of $4 \mathrm{mM}$ AAPH and $20 \mu \mathrm{L}$ of $100 \mathrm{mM}$ CYPMPO, was illuminated for $5 \mathrm{~s}$. Alkyl-oxy radical trapping activity was expressed as an equivalent concentration to GSH (mM/mg sample) (Fig. 1).

Superoxide radical trapping activity Superoxide radical trapping activity was estimated according to the method of Liu et al. (1990). Superoxide radical was generated by HPX/ XOD system. A mixture of $50 \mu \mathrm{L}$ of $4 \mathrm{mM}$ HPX, $30 \mu \mathrm{L}$ of $0.66 \mathrm{mM}$ DTPA, $50 \mu \mathrm{L}$ of sample solution, $20 \mu \mathrm{L}$ of $50 \mathrm{mM}$ CYPMPO and $50 \mu \mathrm{L}$ of XOD $(0.5$ units $/ \mathrm{mL})$ was transferred into a disposable borosilicate ESR flat cell and ESR spectra of superoxide radical and CYPMPO adduct were analyzed. Superoxide radical trapping activity was expressed as SOD equivalent units/g sample.

ESR measurements For ESR measurements, X-band ESR spectrometer (RE-1X and TE-300, JEOL, Akishima, Japan) equipped with $100 \mathrm{kHz}$ field modulation. We employed Win-Rad operation software (Radical Research) for ESR signal acquisition. Typical spectrometer settings were as following: resonance field, $336.2 \mathrm{mT}$; field modulation width, $0.1 \mathrm{mT}$; microwave power, $6 \mathrm{~mW}$; field scan width/rate, \pm 3.5 $\mathrm{mT} / \mathrm{min}$; time constant, $0.1 \mathrm{~s}$. ESR spectra were accumulated at room temperature. 
A typical ESR procedure was as follows. A phosphate buffer solution of AAPH, antioxidant, and CYPMPO was loaded into a disposable borosilicate flat sample cell and placed inside the ESR cavity. In situ illumination was carried out, and the ESR signal was recorded immediately after the illumination was stopped in the case of the alkyl-oxy radical. The superoxide radical was generated by HPX/XOD system. The intensity of the selected ESR line was recorded as a function of radical trapping concentration.

Kinetics We applied the method of Kohri et al. (2009) to analyze our data.

The peak-to-peak intensity of the selected ESR line of the free radical adduct was monitored in the presence or absence of the selected antioxidant (GSH or SOD). In the presence of the spin trap (ST) and antioxidant (AOx), the following free radical $(\mathrm{R})$ trapping reactions should occur:

$$
\begin{aligned}
& \mathrm{R}+\mathrm{ST} \rightarrow \text { R-adduct rate constant: } k_{s t} \\
& \mathrm{R}+\mathrm{AOx} \rightarrow \text { product rate constant: } k_{A O x}
\end{aligned}
$$

When $I_{0}$ and $I$ are the ESR peak heights in the presence of ST alone and ST + AOx, respectively, the amount of the product in Eq. (2) is $I_{0}-I$. Thus, $I$ and $I_{0}-I$ are proportional to $k_{s t}[\mathrm{R}][\mathrm{ST}]$ and $k_{A O x}[\mathrm{R}][\mathrm{AOX}]$, respectively, to give

$$
\left(I_{0}-I\right) I=k_{A O x} /[\mathrm{R}][\mathrm{AOx}] / k_{s t}[\mathrm{R}][\mathrm{ST}]
$$

When nearly negligible portions of ST and AOx are consumed in this reaction, we can safely replace $[\mathrm{ST}]$ and [AOx] with $[\mathrm{ST}]_{0}$ and $[\mathrm{AOx}]_{0}$, respectively, where []$_{0}$ denotes the initial concentration of the component. Eq. (3) can then be rewritten as

$$
I_{0} / I-1=k_{A O x} / k_{s t} \cdot[\mathrm{AOx}]_{0} /[\mathrm{ST}]_{0}
$$

In conclusion, a plot of $I_{0} / I-1$ against $[\mathrm{AOx}]_{0} /[\mathrm{ST}]_{0}$ gives a zero-crossing line with a slope of $k_{A O X} / k_{S t}$. When the same spin trap and free radical-generating condition are used, $k_{s t}$ becomes common for all antioxidants. If $k_{A O x} / k_{s t}$ for each sample is normalized using that of the standard GSH or SOD, the result can be expressed in terms of GSH concentration or SOD units.

\section{Results}

Alkyl-oxy radical trapping activity The ESR spectrum in Fig. 2 demonstrates the sample of CYPMPO and alkyloxy radical adduct. An aqueous solution of AAPH (1 mM) and CYPMPO $(10 \mathrm{mM})$ was illuminated with $\mathrm{Hg}-\mathrm{Xe}$ arc lamp light for $5 \mathrm{~s}$, to obtain a very clear and stable ESR signal of CYPMPO with alkyl-oxy radical adduct.

Figure 3 shows the variation of alkyl-oxy adducts of CYPMPO as a function of the antioxidant GSH concentration. The peak-to-peak intensity of the line marked with an aster-

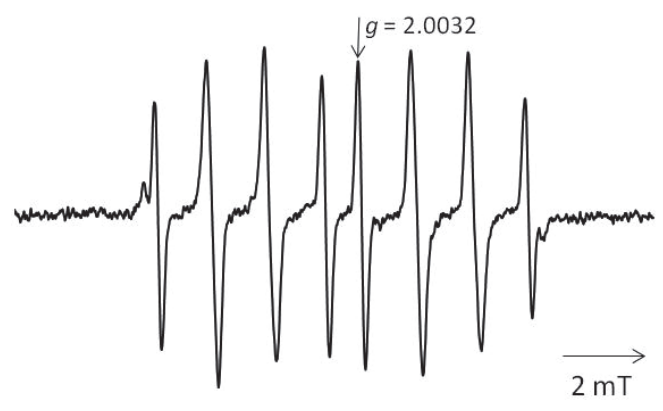

Fig. 2. ESR spectrum of CYPMPO and alkyl-oxy radical adduct. An aqueous solution of AAPH $(1 \mathrm{mM})$ and CYPMPO $(10 \mathrm{mM})$ was illuminated with $\mathrm{Hg}$-Xe arc lamp light for $5 \mathrm{sec}$, and the ESR spectrum was recorded.

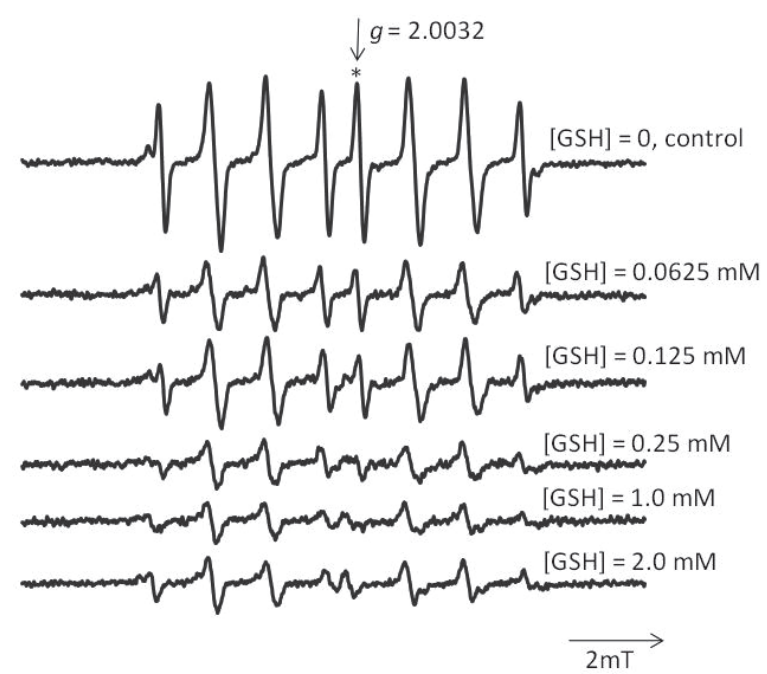

Fig. 3. Change in the level of the alkyl-oxy adduct of CYPMPO as a function of the antioxidant GSH concentration. The peak-to-peak intensity of the line marked with an asterisk $(*)$ was adopted as $I_{0}$ or $I$ to calculate the relative alkyl-oxy spin trap rate $\left(k_{A O x} / k_{S T}\right)$.

isk (*) was adopted as $I_{0}$ or $I$ to calculate the relative alkyloxy radical trapping rate $\left(k_{A O x} / k_{S T}\right)$. The GSH concentration varied from 0 to $2.0 \mathrm{mM}$. To calculate the relative alkyl-oxy radical trapping rate, we used solutions at a concentration of 0 to $0.25 \mathrm{mM}$, as shown in Fig. 4 .

The alkyl-oxy trapping activity of each specimen is shown in Fig. 5. The trapping activity of all specimens was very high. The Chinese crude medicines showed higher abilities than berries. The ethanol extracts of the specimens indicated a higher ability than that of water extracts. As these CYPMPO adducts appeared to be stable, we could calculate the alkyl-oxy radical trapping ability in terms of the GSH concentration.

Superoxide radical trapping activities The ESR spectrum shown in Fig. 6 was determined from the CYPMPO and superoxide radical adduct. An aqueous solution of HPX (1 $\mathrm{mM})$, CYPMPO $(5 \mathrm{mM})$ and XOD $(0.5$ units $/ \mathrm{mL})$ was used for ESR spectroscopy. We obtained CYPMPO and superoxide radical adduct. The CYPMPO adduct was very stable in 


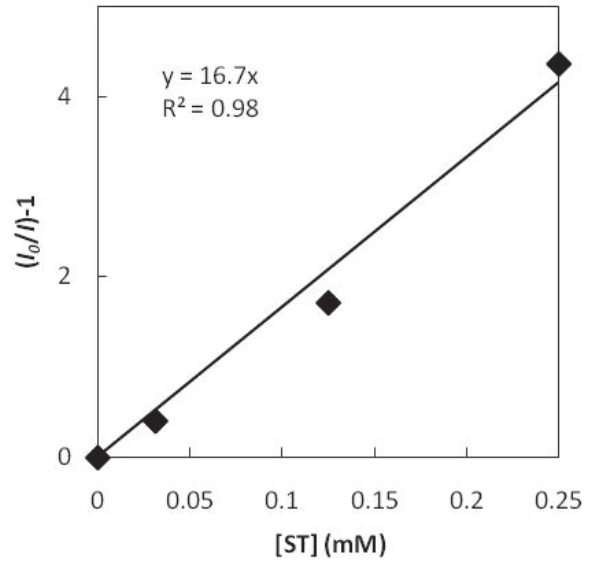

Fig. 4. A plot of $\left(I_{0} / I\right)-1$ versus $[\mathrm{ST}]_{x}$, where $I_{0}$ and $I$ denote ESR intensity in the absence and presence of GSH. [ST] is GSH (mM).

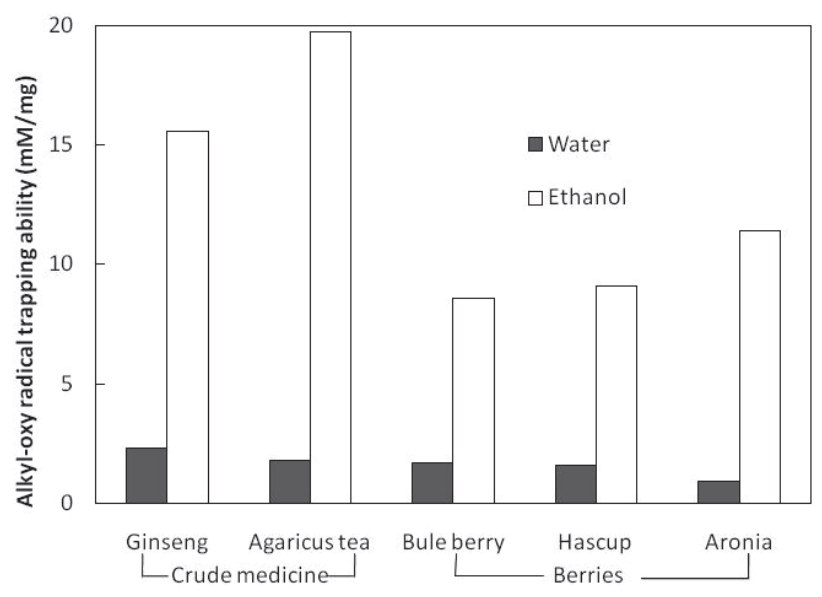

Fig. 5. The alkyl-oxy radical trapping ability of plant extracts. The ability is shown in terms of the GSH concentration ( $\mathrm{mM} / \mathrm{mg}$ ).

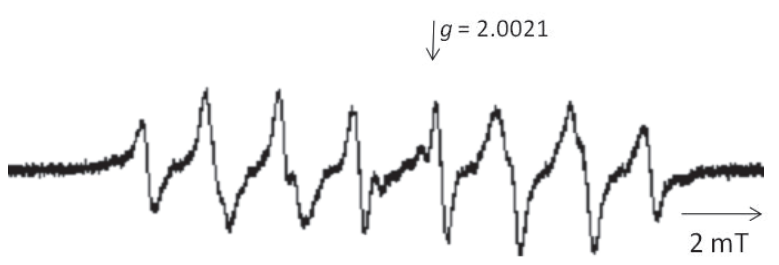

Fig. 6. An ESR spectrum of CYPMPO and superoxide radical adduct. An aqueous solution of HPX (1 mM), CYPMPO (5 mM) and XOD (0.5 units/mL) was used.

HPX-XOD system. The ESR signal of the CYPMPO adduct was detected for 50 min (Kamibayashi et al., 2006). To calculate the relative superoxide radical trapping rate, we used solutions at concentrations of 0 to 5 units, as shown in Fig. 7.

The superoxide radical trapping activity of the specimens is shown in Figs. 8 and 9. The superoxide radical trapping ability of the tea was very high. Japanese basil and burdock, traditional vegetables in Japan, also showed high superoxide

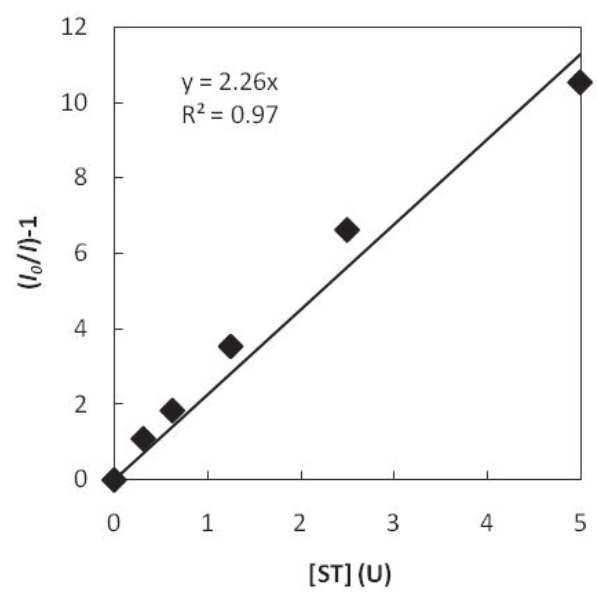

Fig. 7. A plot of $\left(I_{0} / I\right)-1$ versus $[\mathrm{ST}]_{x}$, where $I_{0}$ and $I$ denote ESR intensity in the absence and presence of SOD. [ST] is SOD (unit).

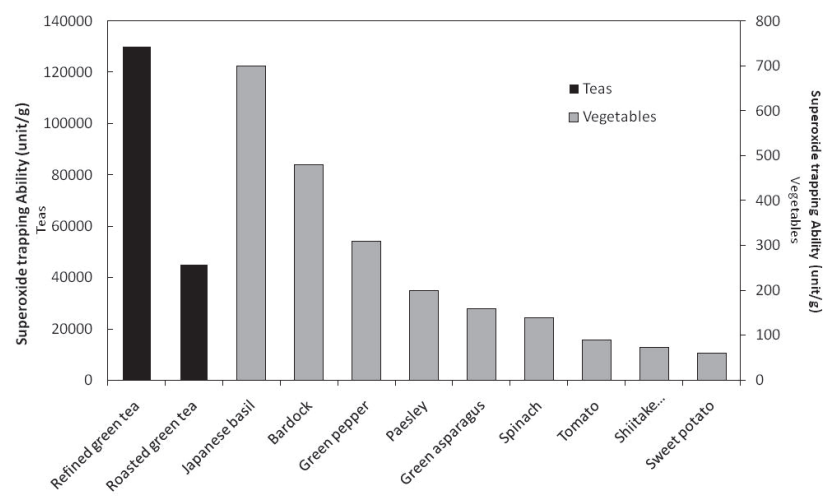

Fig. 8. The superoxide spin trapping ability of water plant extracts. The ability is shown in terms of SOD units (units/g).

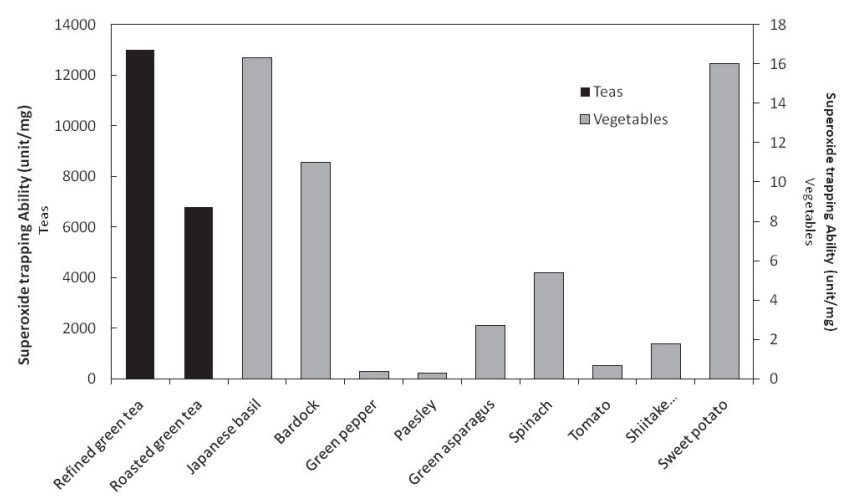

Fig. 9. The superoxide spin trapping ability of $80 \%$ ethanol plant extracts. The ability is shown in terms of SOD units (units/g).

radical trapping activities.

\section{Discussions}

Resolution of trapping method Methods for spin trapping of alkyl-oxy and superoxide radicals were based upon the earlier reports (Rosen et al., 1984; Lagercrantz, 1975; Janzen, 1971; Janzen, 1984). In the present study, the relative free radical trapping activities of various plant foods were 
evaluated based on normalizing the ESR signals relative to the standard activity of GSH as a scavenger of alkyl-oxy radicals and SOD as a superoxide scavenger. We concluded that CYPMPO has a high capability for radical trapping and is useful for the analysis of the plant radical system.

Antioxidant activities Comparisons of activities showed that the crude medicine extracts with ethanol had strong alkyl-oxy radical trapping activities. Berries extracts with ethanol had also strong alkyl-oxy radical trapping activity. The extracts with water showed almost same alkyl-oxy radical trapping activities.

Tea extracts with water and ethanol showed very high superoxide trapping abilities. Refined green teas showed higher ability than roasted green teas. More than half of the activity was lost after roasting treatment. It was suggested that for superoxide trapping activity, much of the activity was affected by temperature.

Solubility of the components of crude plant extracts is also important. Radical trapping activities reported here only refers to the soluble components either water or ethanol, or of those substances that have passed through paper filter. Hence most of the contribution towards radical trapping assayed from extracts is by low molecular weight materials.

Future scope We plan to examine the antioxidant activities of other botanical substances and herbs. The use of treatments such as heat and filtration may help clarify the relative contribution of components to radical trapping activity. Furthermore, trapping of other radical species can be assayed by CYPMPO.

Acknowledgements We are grateful for the "Basic research on the utilization of radiation for food expected on practical application" grant under the Strategic Promotion Program for Basic Nuclear Research by the Ministry of Education, Culture, Sports, Science and Technology of Japan.

\section{Appendix}

CYPMPO: 5-(2,2-dimethy-1,3-propoxy-cyclophosphoryl)

-5-methyl-1-pyrroline $\mathrm{N}$-oxide),

AAPH: 2,2'-azobis(2-amidinopropane) dihydrochloride

HPX: hypoxanthine

DTPA: diethylene-triamine-pentaacetic acid

XOD: xanthine oxidase

GSH: 2-amino-5-\{[2-[(carboxymethyl)amino]-

1-(mercaptomethyl)-2-oxoethyl]amino -5-oxopentanoic acid

SOD: superoxide dismutase

ORAC: Oxygen Radical Absorption Capacity

Trolox: 6-hydroxy-2,5,7,8-tetramethylchroman-2-carboxylic acid
ABTS: 2,2'-azinobis(3-ethylbenzthiazoline-6-sulphonate

DPPH: 1,1-diphenyl-2-picrylhydrazyl

\section{References}

Delincee, H. (2002). Improvement of the ESR detection of irradiated food containing cellulose employing a simple extraction method. Radiat. Phys. Chem., 63, 455-458.

Exarchou, V., Nenadis, N., Tsimidou, M. and Agric, J. (2002). Antioxidant activities and phenolic composition of extracts from Greek oregano, Greek sage, and summer savory. Food Chem., 50, 5294-5299.

Horváthová, J., Suhaj, M., Polovka, M., Brezová, V. and Šimko, P. (2007). The influence of gamma-irradiation on the formation of free radicals and antioxidant status of oregano. Czech J. Food Sci., 25, 131-143.

Janzen, E.G. (1971). Spin trapping. Chem. Res., 4, 31-40. Janzen, E.G. (1984). Spin trapping. Meth. Enzymol., 105, 188-198. Jun, W.J., Han, B.K. and Yu, K.W. (2001). Antioxidant effects of Origanum majorana L. on superoxide anion radicals. Food Chem., 75, 439-444.

Kamibayashi, M., Oowada, S., Kameda, H., Okada, T., Inanami, O., Ohta, S., Ozawa, T., Makino, K. and Kotake, Y. (2006). Synthesis and characterization of a practically useful DEPMPOtype spin trap, 5-(2,2-dimethyl-1,3-propoxy cyclophosphoryl)-5methyl -1-pyrroline $N$-oxide (CYPMPO). Free Radic. Res., 40, 1166-1172.

Kikuzaki, H., Kawasaki, Y. and Nakatani, N. (1994). Structure of antioxidative compounds in ginger. Am. Chem. Soc., 547, 237-243.

Kohri, S., Fujii, H., Oowada, S., Endoh, N., Sueishi, Y., Kusakabe, M., Shimmei, M. and Kotake, Y. (2009). An oxygen radical absorbance capacity-like assay that directly quantifies the antioxidant's scavenging capacity against AAPH-derived free radicals. Analytical Biochem., 386, 167-171.

Krainev, A. and Bigelow, D.J. (1996). Comparison of 2,2'-azobis (2-amidinopropane) hydrochloride (AAPH) and 2,2'-azobis (2,4-dimethylvaleronitrile) (AMVN) as free radical initiators: a spin-trapping study. J. Chem. Soc. Perkin Trans., 2, 747-754.

Lagercrantz, C. (1975). Spin trapping of some short-lived radicals by the nitroxide method. J. Phys. Chem., 75, 3466-3475.

Liu, J., Edamatsu, R., Kabuto, H. and Mori, A. (1990). Antioxidant action of guilingji in the brain of rats with $\mathrm{FeCl} 3$-induced epilepsy. Free Radic. Biol. Med., 9, 451-454.

Martinéz-Tomé, M., Jiménez, A.M., Ruggieri, S., Frega, N., Strabbioli, R. and Murcia, M.A. (2001). Antioxidant Properties of Mediterranean Spices Compared with Common Food Additives. J. Food Prot., 64, 1412-1419.

Moreno-Sánches, C. (2002). Methods used to evaluate the radical scavenging activity in foods and biological systems. Food Sci. Tech. Int., 8, 121-137. 
Nakamura, H., Ukai, M. and Shimoyama, Y. (2006). An electron spin resonance study of ginseng irradiated by gamma ray. Spectrochemica Acta, 63, 883-887.

Percival, M. (1996). Antioxidants. Clin. Nutr. Ins., 1, 1-6.

Polovka, M., Brezová, V. and Šimko, P. (2007). A tool to characterise the gamma irradiated foods. J. Food Nutr. Res., 46, 75-83.

Prior, R.L., Hoang, H., Gu, L., Bacchiocca, M., Howard, L., Hampsch-Woodill, M., Huang, D., Ou, B. and Jacob, R. (2003). Assays for hydrophilic and lipophilic antioxidant capacity (Oxygen Radical Absorbance Capacity (ORAC)) of plasma and other biological and food samples. J. Agric. Food Chem., 51, 3273-3279.

Prior, R.L., Wu, X. and Schaich, K. (2005). Standardized methods for the determination of antioxidant capacity and phenolics in foods and dietary supplements. J. Agric. Food Chem., 53, 3101-3113.

Rosen, G.M. and Rauckman, E.J. (1984). Spin trapping of superoxide and hydroxyl radicals. Meth. Enzymol., 105, 198-209.

Suh, H.J., Lee, J.M., Kim, Z.S. and Chung, S.H. (1996). Radical scavenging compound in onion skin. Food Res. Int., 32, 659-664.

Suhaj, M., Rácová, J., Polovka, M. and Brezová, V. (2006). Effect of gamma-irradiation on antioxidant activity of black pepper (Piper nigrum L.). Food Chem., 97, 696-704.
Suhaj, M. (2006). Spice antioxidants isolation and their antiradical activity: a review. J. Food Compos. Anal., 19, 531-537.

Ukai, M. and Shimoyama, Y. (2003). Free radicals in irradiated pepper: an electron spin resonance study. Appl. Magn. Reson., 24, $1-11$.

Ukai, M., Kameya, H., Nakamura, H. and Shimoyama, Y. (2008). An electron spin resonance study of dry vegetables before and after irradiation. Spectrochemica Acta, 69, 1417-1422.

Wang, M., Jiangang, H., Rangarajan, M., Shao, Y., LaVois, E.J., Hung, T.C. and Ho C.T. (1998). Antioxidative phenolic compounds from sage (Salvia officinalis). J. Agric. Food Chem., 46, 4869-4873.

Yang, G.C., Zasaei, P.M. and Page, S.W. (1994). Garlic as antioxidants and free radical scavengers. J. Food Drug Anal., 1, 357-364.

Yordanov, N.D. and Aleksieva, K. (2004). X- and Q-band EPR studies on fine powders of irradiated plants. New approach for detection of their radiation history by using Q-band EPR spectrometry. Radiat. Phys. Chem., 69, 59-64.

Zheng, W. and Wang, S.Y. (2001). Antioxidant activity and phenolic compounds in selected herbs. J. Agric. Food Chem., 49 , 5165-5170. 